\title{
Full Wave Simulation and Design of Film Bulk Acoustic Wave Filters
}

\author{
Du Bo ${ }^{1,2}$, Lin Jian², Ou Li' ${ }^{2}$ Shi Yu' and MA Jin-yi ${ }^{2}$ \\ ${ }^{1}$ State Key Lab.of Electronic Thin Films and Integrated Devices, University of Electronic Science and Technology of China, 610054 \\ Chengdu, China
}

${ }^{2} 26$ th Institude of China Electronics Technology Group Corporation, 400060 Chongqing, China

\begin{abstract}
In order to simulate the frequency response of thin film bulk acoustic wave filter accurately, the MBVD model of film bulk acoustic wave filter chip and three dimension electro-magnetic model of package were respectively established. And then, the collaborative simulation of acoustic and electro-magnetic components was realized in ADS software. Finally, thin film bulk acoustic wave filter with working frequency of $2.825 \mathrm{GHz}$ and 5.6 $\mathrm{GHz}$ were designed and fabricated by this method. Insertion loss of $2.825 \mathrm{GHz}$ FBAR filter is less than $1.5 \mathrm{~dB}$, and rejection is more than $40 \mathrm{~dB}$. Insertion loss of $5.6 \mathrm{GHz}$ FBAR filter is less than $1.7 \mathrm{~dB}$, and rejection is more than $30 \mathrm{~dB}$. The simulation and experimental results show that the proposed method has good design precision and can simulate the influence of package on the performance of the filter accurately.
\end{abstract}

\section{Introduction}

Thin film bulk acoustic wave resonator (FBAR) filter has the advantages of higher operating frequency, smaller size, higher Q value and better power handling capability, comparing surface acoustic wave (SAW) filter, LC filter and ceramic filter [1]. Furthermore, its process is compatible with integration circuit (IC) process [2]. So, FBAR filter has been used widely in mobile communication market.

FBAR utilizes the longitudinal wave resonate characteristics of piezoelectric materials such as AlN or $\mathrm{ZnO}$ to realize electrical frequency selection $[3,4]$. Acoustic waves are about five orders of magnitude shorter than electromagnetic waves, resulting in significantly small size. FBAR filter is combined by multiple FBAR resonators in a certain circuit structure, including ladder structure and bridge structure [5]. Ladder structure is often used in single ended circuit, and the bridge structure is used in balance ended circuit structure.

The frequency of FBAR is mainly determined by the acoustic velocity and thickness of piezoelectric film. The acoustic velocity of AIN is about $11000 \mathrm{~m} / \mathrm{s}$, and the thickness of AlN can be controlled between 0.1 micros to 2 micros. So, FBAR can work at the frequency of 1 to 10 $\mathrm{GHz}[6]$. At this high working frequency, the parasitic parameters of the package have significant influence on the frequency response of the filter. Therefore, in order to realize more accuracy design, the effect of package should be considered in the FBAR filter simulation model.

The FBAR filter is made by MEMS process, and it is based on Microwave acoustic theory. While the ceramic package is made of HTCC process and its influence on the filter is based on electro-magnetic wave theory. How to realize co-simulation of acoustic and electro-magnetic components in the design process is difficult.

Traditional design method is to fit the equivalent circuit parameters of package by measuring the frequency response of the packaged filter, and then use the equivalent circuit for the filter design [7]. The drawback of this approach is that in order to get accurate parameters, it needs to produce and measuring the device multi-times. Meanwhile, if the package or bonding method is changed, it needs to fit the measurement results again. So, its efficiency is low.

This paper used the ADS software to set up a FBAR MBVD (Modified Butterworth-Van Dyke) equivalent circuit model for the simulation of filter chip. The three dimension (3D) electro-magnetic model of ceramic package was established with HFSS software. And then, the $S$ parameters of package was achieved and added into FBAR filter MBVD model. This method combined electro-magnetic and acoustic simulation, called full wave simulation. Using this method, FBAR filters working at $2.825 \mathrm{GHz}$ and $5.6 \mathrm{GHz}$ was designed, produced and measured.

\section{Full wave simulations}

\subsection{FBAR equivalent circuit model}

In order to realize the fast and accurate design of FBAR filter, MBVD model is usually adopted. The equivalent circuit model use lumped elements to describe FBAR 
resonator, so it is conducive to the rapid optimization of filter frequency response [8].

Fig. 1 is MBVD model of FBAR resonator, comprising 6 lumped parameter components. The dynamic impedance is on behalf of electrical and mechanical response of piezoelectric materials characterization, including dynamic capacitance $\mathrm{C}_{\mathrm{m}}$, dynamic inductance $\mathrm{L}_{\mathrm{m}}$ and resistance $\mathrm{R}_{\mathrm{m}}$. Capacitance $\mathrm{C}_{0}$ represents the dielectric properties of piezoelectric material. $\mathrm{R}_{0}$ represents the dielectric loss of piezoelectric materials and related mechanical vibration loss. $R_{s}$ is from the ohmic loss of electrodes.

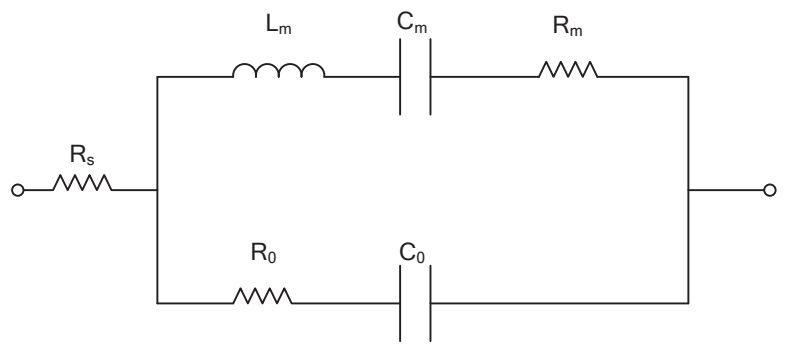

Figure 1. MBVD equivalent circuit model.

FBAR resonator needs be fabricated and measured, and the MBVD model parameters of FBAR resonator can be accurately extracted by fitting measured $S$ parameters. The measurement and fitted Smith circle of FBAR are shown in Fig.2. The MBVD model fits well with measured data above resonator frequency (fs). However, because of parasitic resonances caused by RayleighLamb (RL) modes, it can't fit the measured data below fs. Nevertheless, the precision of MBVD model is good enough for filter simulation.

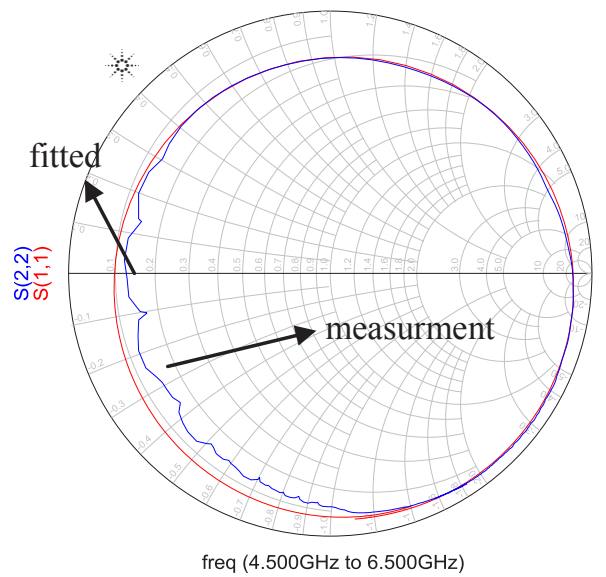

Figure 2. Measured and fitted Smith circle of FBAR.

According to the ladder or bridge circuit structure, as shown in Fig.3, the MBVD models of each resonator are combined, and then the equivalent circuit simulation model of the filter is constructed. In order to realize a flat passband, the anti-resonant frequency of parallel resonators is approximately equal to the resonant frequency of series resonators. Generally, the frequency of parallel resonators is $6 \% \sim 8 \%$ lower than that of series resonators.

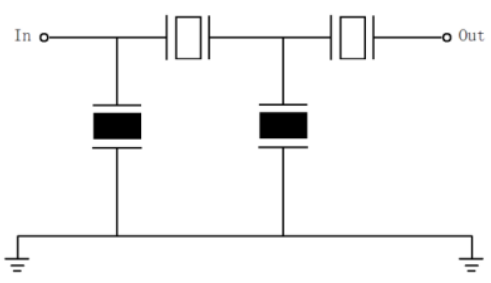

(a) Ladder type

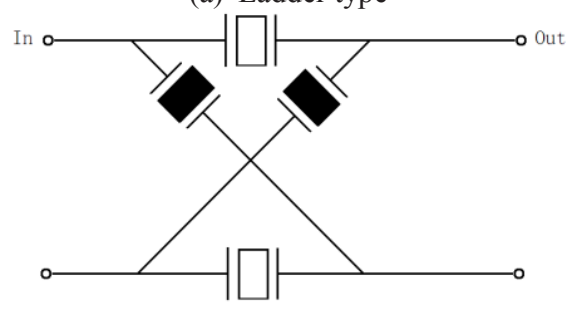

(b) Bridge type

Figure 3. FBAR filter circuit structures.

The ladder structure is single ended structure, which is widely used. It can realize good rectangle coefficient, but its rejection is poor, so it is necessary to introduce inductors in the parallel resonators to improve the out-ofband rejection characteristics. Fig. 4 is the admittance of resonator series with and without inductor. As shown, the introduction of inductor can cause the series resonant frequency of resonator to move to lower frequency. Therefore, different inductors can produce several zero points, and the rejection of filter can be improved.

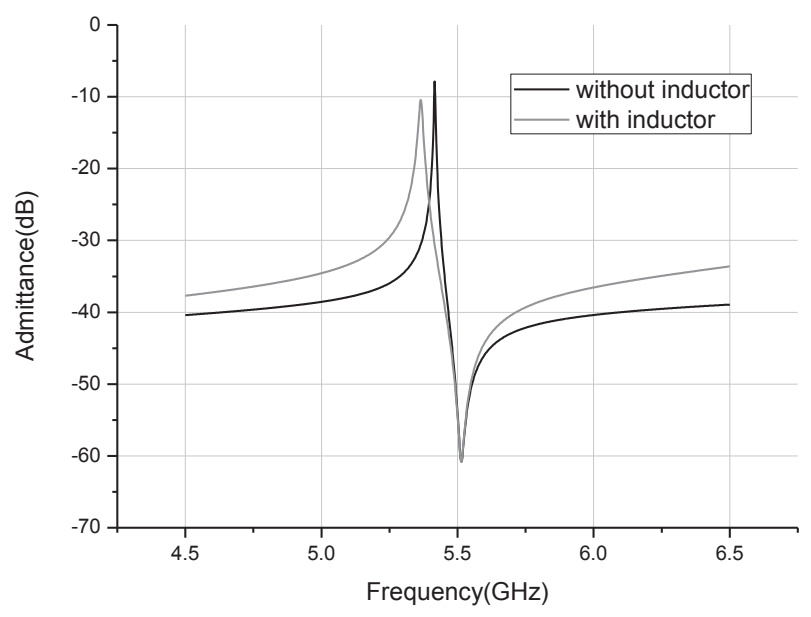

Figure 4. The admittance of resonator series with and without inductor.

\subsection{The influence of package to filter frequency response}

FBAR filter has high working frequency between $1 \mathrm{GHz}$ to $10 \mathrm{GHz}$. At this high frequency, the inductors series with the parallel resonators are usually realized by parasitic inductance of package, such as bonding wire, 
embedded inductor and so on. At the same time, the parasitic capacitance of the package also has significant influence on the out-of-band rejection of the filter.

Fig. 5 and Fig. 6 show the measured frequency responses of FBAR filters working at $1.56 \mathrm{GHz}$, packaged with different ceramic envelopes. It's clearly that the frequency responses of these filters are different, especially for out-of-band rejection characteristics.

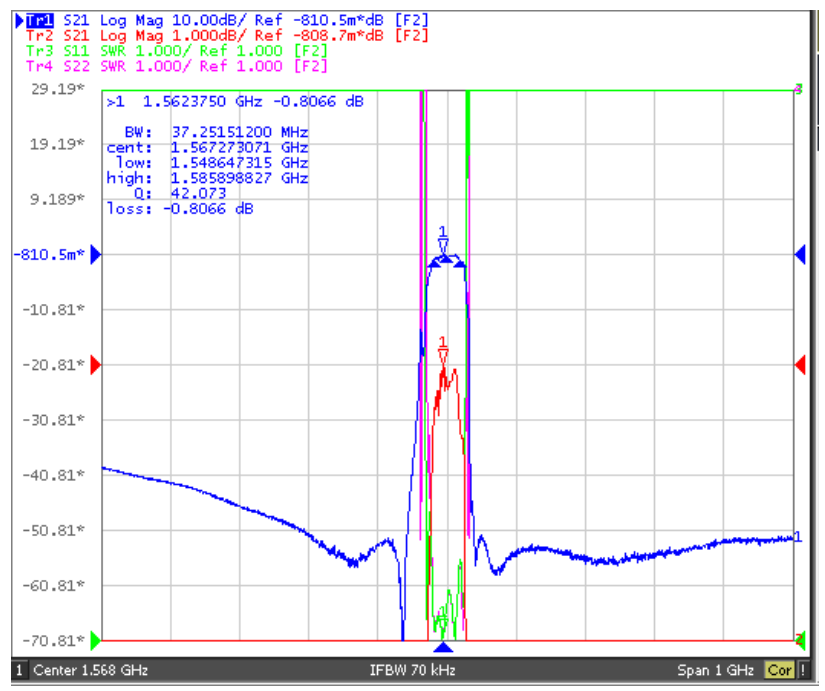

Figure 5. FBAR filter packaged with ceramic envelope A.

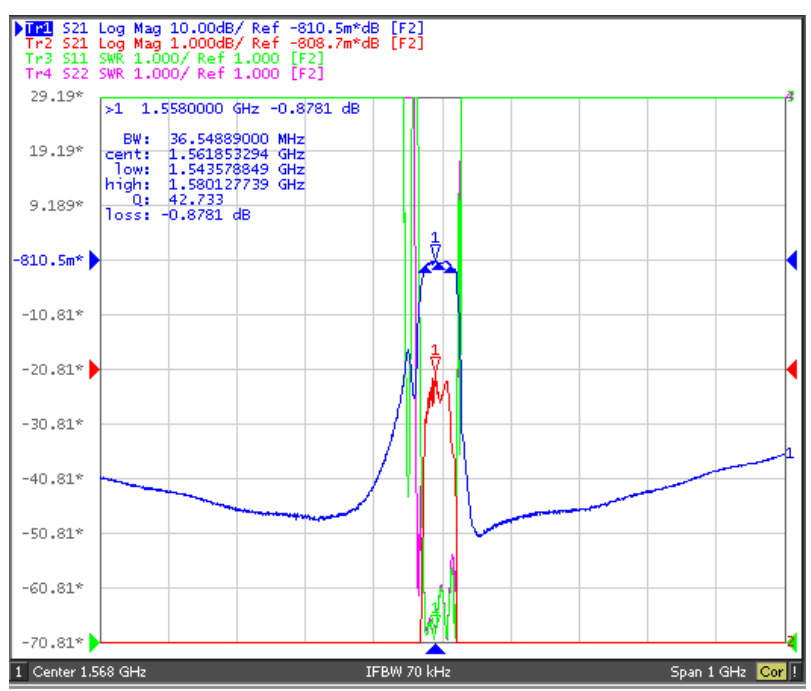

Figure 6. FBAR filter packaged with ceramic envelope B.

During the process of design FBAR filer chip, if not considering the influence of package, the measured results will be different with simulations.

\subsection{D electromagnetism model of FBAR package}

In order to extract the electromagnetic parameters of package accurately, 3D electro-magnetic model of ceramic package was established by electro-magnetic simulation software HFSS, as shown in Figure 7. And the 8 ports $\mathrm{S}$ parameters were calculated and extracted. Inside the model, two ports were set as input and output ports of package, and the other six ports were set as interconnecting ports of the package and the filter chip.

The model includes ceramic package, bonding wire, chip substrate, bonding pad and so on. The size of ceramic package is $3 \mathrm{~mm} \times 3 \mathrm{~mm} \times 1.1 \mathrm{~mm}$, the bonding wires are gold wire. The chip substrate material is high resistance silicon, and the bonding pad material is gold film. The chip size is $1 \mathrm{~mm} \times 1 \mathrm{~mm} \times 0.35 \mathrm{~mm}$.

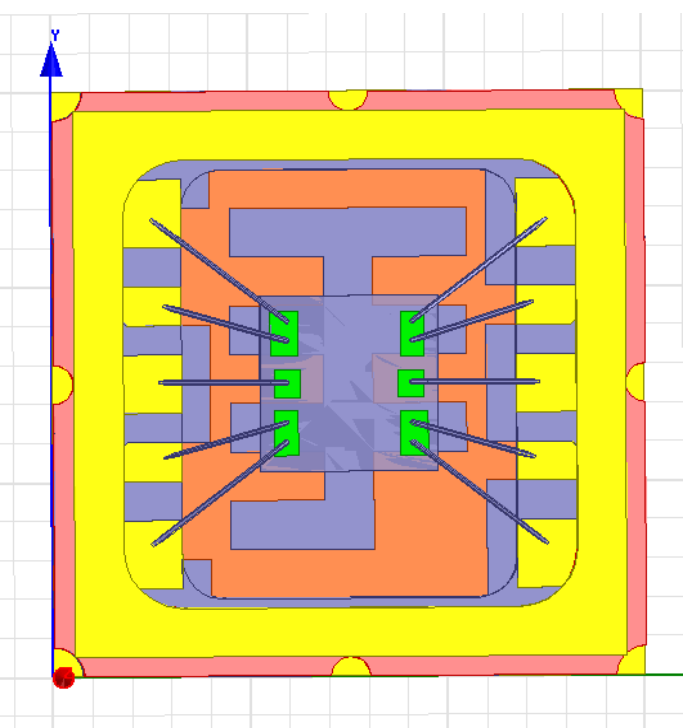

Figure 7. 3D electromagnetism model of FBAR package.

\subsection{Full wave simulation of FBAR filter}

ADS software was used to realize full wave simulation of FBAR filter. S parameters of package was introduced into ADS, and connected with MBVD model of FBAR filter chip. Input and output ports of package were connected with terminals of S-parameter simulators, which are used to define the impedance and location of the ports, as shown in Fig 8. After building the model, the resonator areas of filter were tuned to optimize the filter frequency response. If the frequency response can't meet the specification requirements, it is necessary to refine the bonding method or replace the package.

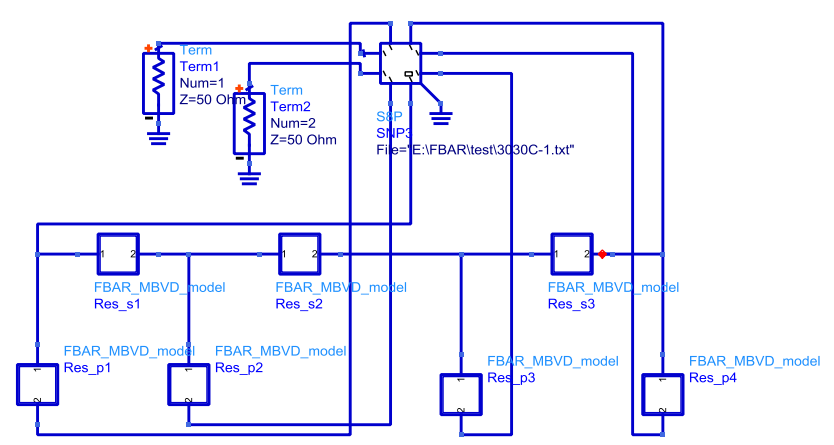

Figure 8. Full wave simulation model of FBAR filter. 


\section{Fabrication and measurement of FBAR filters}

Using full wave simulation method, the FBAR filters working at $\mathrm{S}$ band and $\mathrm{C}$ band are optimized and fabricated and measured.

\section{1. $S$ band FBAR filter}

Fig. 9 is $\mathrm{S}$ band FBAR filter chip photograph, and Fig. 10 is comparison of simulation result of $\mathrm{S}$ band FBAR filter with measurement result. The central frequency of the device is $2.825 \mathrm{GHz}$, the insertion loss is $1.5 \mathrm{~dB}$, and the out of band rejection is more than $40 \mathrm{~dB}$.

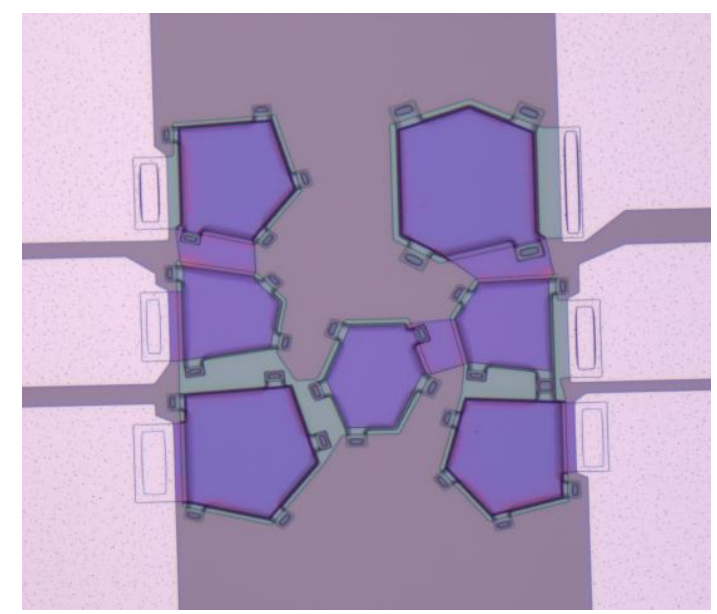

Figure 9. S band FBAR filter chip photograph.

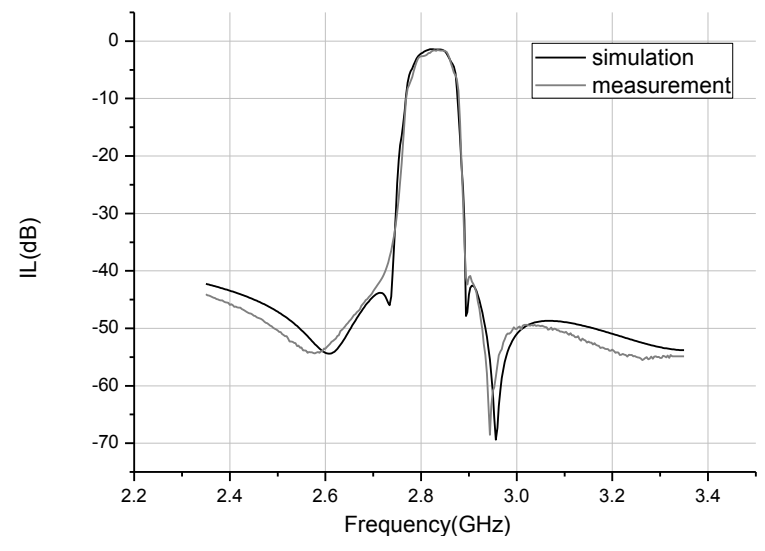

Figure 10. Comparison of simulation result of $\mathrm{S}$ band FBAR filter with measurement result.

\subsection{C band FBAR filter}

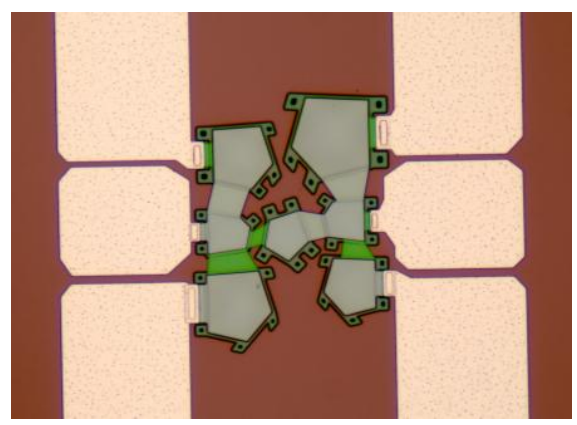

Figure 11. $\mathrm{C}$ band FBAR filter chip photograph.

Fig. 11 is $\mathrm{C}$ band FBAR filter chip photograph, and Fig. 12 is comparison of simulation result of $\mathrm{C}$ band FBAR filter with measurement result. The central frequency of the device is $5.6 \mathrm{GHz}$, the insertion loss is $1.7 \mathrm{~dB}$, and the out of band rejection is more than $30 \mathrm{~dB}$.

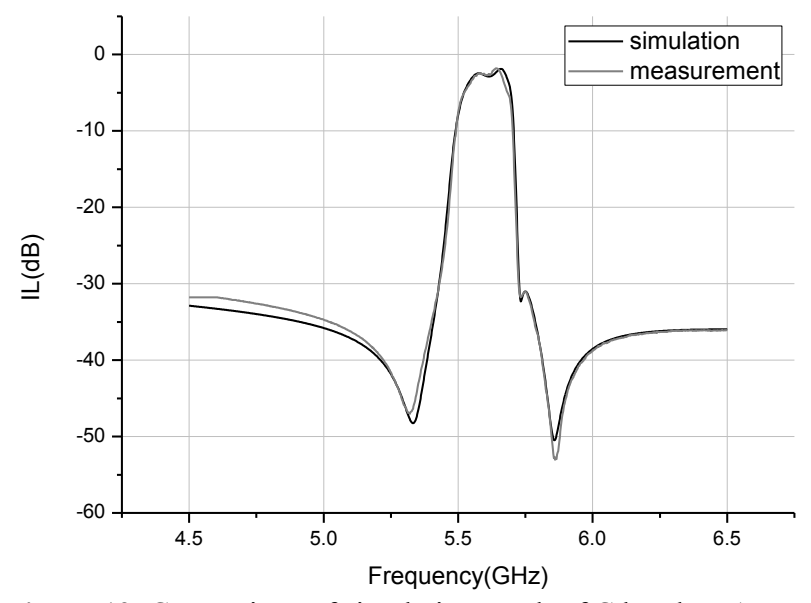

Figure 12. Comparison of simulation result of $\mathrm{C}$ band FBAR filter with measurement result.

All those results show that the simulation results of $\mathrm{S}$ and $\mathrm{C}$ band filters are in good agreement with the measured results.

\section{Conclusions}

The FBAR filter operates at very high frequencies and covers $1-10 \mathrm{GHz}$. In such a high frequency, the influence of parasitic parameters of bonding wire and packages to the frequency responses of FBAR filter is significant. If not considering the influence of package in the filter design process, it is likely to lead to deterioration in the frequency responses of FBAR filter after filter chip packaged.

However, how to realize co-simulation of acoustic and electro-magnetic components in the design process is difficult. This paper used full wave design method, combined electro-magnetic and acoustic models.

With this design method, the optimized design of FBAR filter with $2.825 \mathrm{GHz}$ and $5.6 \mathrm{GHz}$ working frequency were completed. Insertion loss of $2.825 \mathrm{GHz}$ FBAR filter is less than $1.5 \mathrm{~dB}$, and rejection is more than $40 \mathrm{~dB}$, and insertion loss of $5.6 \mathrm{GHz}$ FBAR filter is less than $1.7 \mathrm{~dB}$, and rejection is more than $30 \mathrm{~dB}$. 
The simulation and measurement results show that this design method has good simulation accuracy, and can be used for FBAR design.

\section{References}

1. J. S. Shin, Y.K. Park, Y.I. Kim et al, Proceedings of the 40th European Microwave Conference, 12571260 (2010)

2. M. Aissi, E. Tournier, M.A. Dubois, et al, IEEE International Solid-State Circuits Conference, 17.5 (2006)

3. F. Martin, M.-E. Jan, B. Belgacem, M.-A. Dubois, P. Muralt, Thin Solid Films, 514, 341-343 (2006)
4. L. Mai, J.Y. Lee, V.S. Pham, and G.Y, IEEE Microwave and Wireless Components Letters, 17, 12, (2007)

5. Uzunov, D. Gajdajiev, MIXDES 2011, 18th International Conference "Mixed Design of Integrated Circuits and Systems", 16-18 (2011)

6. M. Hara, T. Yokoyama, M. Ueda and Y. Satoh, IEEE Ultrasonic Symposium, 1152-1155 (2007)

7. F. M. Pitschi, J. E. Kiwitt, B. Bader, and K. C. Wagner, IEEE International Ultrasonics, Ferroelectrics, and Frequency Control Joint 50th Anniversary Conference, 1525-1528 (2004)

8. J. D. Larson, SM, P.D. Bradley, et al, IEEE Ultrasonic Symposium, 863-868 (2000) 